\title{
The Development of Zheng Practice Program Based on Self-regulated Learning Strategies
}

\author{
Xinning $\mathrm{Wu}^{1}$, Yong-Chil Yang ${ }^{2}$ \\ ${ }^{1}$ Andong National University, Andong, South Korea. \\ 2 Andong National University, Andong, South Korea. \\ Correspondence: Yong-Chil Yang, Andiong National University, Andong, Kyungbuk 750-749, Korea.
}

Received: February 10, 2015

Accepted: February 26, 2015

Available online: March 24, 2015

doi:10.11114/ijsss.v3i3.741

URL: http://dx.doi.org/10.11114/ijsss.v3i3.741

\begin{abstract}
An effective music practice of a musical instrument can be attained over the use of self-regulated learning strategies. Musicians often use self-regulated learning strategies in their practice sessions. Similarly, a string instrument can be linked with self-regulated learning, when it comes to its posture, tone production, bowing, and fingering features with various tuning potentials. In our study, we discussed the use of a string instrument for self-regulated learning. We developed a practice program for a string instrument called Zheng in three steps. In the first step, self-regulated learning strategies for the Zheng practice program were derived by surveying the existing literature. In the second step, the Zheng practice model was developed. Finally, the 20 hours Zheng practice program was developed by means of self-regulated learning strategies. The outcomes of the program can be useful for novice learners who are interested to improve their string instrument practice.
\end{abstract}

Keywords: Zheng practice, Self-regulated learning strategies, Instructional design

\section{Introduction}

It is obvious that an effective practice is an essential in the musical instrument learning. Music practice is vital to student musicians in attaining the highest level of performance skills (Chung, 2006; Leon-Guerrero, 2008). Generally, the performance of a musical instrument is required a great deal of time and effort. Expert musicians are more likely to monitor and control their performance by focusing their attention on what they are practicing and how it can be improved (Ericsson et al., 1993). The time amount and the method of the practice are the most important factors for novice musicians to be an expert musician (Nielsen, 1999; Hallam, 2001).

The Zheng instrument is a popular traditional Chinese string instrument which is composed of anhemitonic pentatonic scale holding a unique physical feature and indefinite tuning potentials (Sheng \& Samuel, 2005). Previous studies have focused on developing effective Zheng practice strategies to help learners practice with more effective (Fu, 2004; Lin, 2008; Mao, 2007). However, Zhou (2007) found strategic problems in the Zheng performance and practice. The problems include the size of segments when played without interruption and separation of hands, and ineffective repetitive practice. One way to solve them is to embed self-regulated learning strategies into the Zheng practice programs (Zimmerman, 1986).

Self-regulated learning (SRL) refers to self-generated thoughts, feelings, and behaviors that aim to achieve learning goals and enable students to utilize their metacognitive, motivational and behavioral participation in the learning phase (Zimmerman, 2000). SRL depends above all upon learners' intrinsic motivation in the learning process (Zimmerman \& Martinez-Pons, 1986). The uses of SRL strategies which can enable learners to actively participate in the learning process are required in the music practice and performance (McPherson \& Zimmerman, 2002).

Researchers have found a relationship between diverse practice strategies and SRL components in which novice musicians set a goal to learn new challenging tasks and use problem-solving skills to adjust their practice strategies (Nielsen, 1999; Hallam, 2001; Pitts et al., 2000; Smith, 2002). They have also revealed that novice musicians have a lack of skills to demonstrate SRL strategies during their practice. Recent studies have revealed that SRL strategies could be related to the level of performance in music practice (Chung, 2006; Leon-Guerrero, 2008; McPherson \& Renwick, 2006; Hallam, 2001; Nielsen, 2001; Pitts, 2000; Susan, 2008). However, there have been some problems in these 
previous studies for musical instrument practice based on SRL. First, most studies just investigated the relationship between musical instrument practice and SRL, also just conducted the examination of SRL strategies during musical instrument practice. Second, most studies were mainly conducted regarding Keyboard musical instrument with middle-level participants. Third, little is known about self-regulated learning strategies related to the string instrument practice program through the systematic instructional design. And finally, empirical research on the effects of SRL strategies in music instrument practice has little been conducted.

Thus, in this study, we developed a program of Zheng practice for novices by using self-regulated learning strategies. The study was composed of three steps. The first step was to derive self-regulated learning strategies for the Zheng Practice Program by a literature review. The second step was to make the Zheng practice model. The final step was to develop 20 hour Zheng practice programs by means of self-regulated learning strategies.

\subsection{Music Instrument Practice and Self-Regulated Learning Strategies}

In recent years, researchers have studied how music instrument learners acquire and refine their skills. Some researchers state that the quality of performance is directly related to quantity of deliberate practice (e.g., McPherson, \& Renwick, 2001; Williamon \& Valentine, 2000), many experts have studied the nature of expertise (Hallam, 2001; Pitts \& McPherson, 2000). Research on instrumental practice has mainly concentrated on revealing relationships between learning activities and results. Yet, there has been little known about how novice music performers become experts or what students specifically learn through practice. McPherson and Zimmerman (2002) argued that learning a musical instrument requires a great deal of self-regulation which may influence the participation of students in their learning process. It is evident when students become metacognitively, motivationally, and behaviorally active participants in their own learning process.

SRL strategies have an important role in students' learning processes and performance. They are involved in self-regulated strategies such as self-evaluation, organization and transformation, goal setting and planning, information seeking, record keeping, self-monitoring, environmental structuring, giving self-consequences, rehearsing and memorizing, seeking social assistance, and reviewing (Zimmerman \& Martinez-Pons, 1986). Several studies have explored various principles of SRL strategies to address how students learn to master a given musical performance. They have examined self-regulation strategies used in instrument practice and how it could be related to the performance of instrumental learning (Chaffin \& Imreh, 2001; Hallam, 2001; Kim, 2009). They have examined the relationship between individual practice in music instrumentation and SRL in three forms that include metacognitive regulation, motivational regulation, and behavioral regulation strategies.

Research indicates that expert music performers utilize substantial planning and a systematic approach to practice sessions, including problem identification, strategic planning and evaluation (Hallam, 2001). Sunsan (2008) studied that how college music students of different ages used self-regulated learning behaviors during practice sessions followed by their daily learning. It found that SRL in instrumental practice can be more effective when students organize their practice sessions by setting proximal goals, applying appropriate practice strategies, monitoring their playing, and self-reflecting after their practice sessions. Leon-Guerrero (2008) found that repetition practice of self-regulating strategies was used more frequently than other strategies during music practice of novice instrumental musicians. The research also found that the repetitive practicing strategy used in piano practice of middle-school students (Kim, 2009). However, the traditional assessment in music education courses has properly considered the students' ability to obvious activities, such as executing correct pitches, rhythm, articulations, and dynamic markings. Miksza (2007) showed that the difference between participants' self-evaluation of practice efficiency and performance achievement produced higher with the passage of time. She also found a positive relationship between performance achievement and the practice behaviors in repeat section, whole-part-whole, marks part, and varying pitch.

An effective practice model is suggested in several studies in which students need to deal with the cognitive, technical and emotional aspects of diverse strategies in order to achieve higher levels of performance (Nielsen, 2004; Pitts \& McPherson, 2000). For example, Nielsen (2004) indicated that music performers used considerable planning and reasoning, and anticipation to achieve greater performance. Similarly, Kim (2009) also found that the cognitive regulation was used in the repetitive practicing strategy, self- efficacious ability of the motive regulation of self-performance, achievement value, behavioral regulation in the planning on practicing time and assistance during the time practicing. However, McPherson and Renwick (2006) revealed that students demonstrated little planning and strategies during their actual practice sessions. The studies were to find what self-regulating strategies for aiding in a better understanding of the self-regulation capabilities of student musicians. Too often students are given the assignment to practice during learning an instrument without the understanding of how to organize, plan, monitor and evaluate their progress while working alone.

Previous studies mainly examined cognitive dimensions based on the assessment of students' musical understanding to 
identify their rationales in participation in learning activities through observation of practice behavior, concurrent verbal reports during practice sessions. Specifically, the behavior and verbal data were examined in reference to these self-regulation strategies. Furthermore, it is often that students are given the assignment to practice during learning an instrument without the understanding of how to organize, plan, monitor and evaluate their progress while working alone. Students are rarely provided guidance on how to approach individual practice to gain the level of success that will able them to reach their potential.

\subsection{Music Instrument Practice and the Zheng Practice}

Among various music instruments, a string instrument has more relevance to self-regulated learning when it comes to its nomenclature, posture, tone production, bowing, and fingering features and various tuning potentials like Zheng which is composed of anhemitonic pentatonic scale with unique physical features and indefinite tuning potentials. Zheng is one of the most popular Chinese traditional string instruments. It is different from keyboard instruments. The modern Zheng is a long zither with 21 strings. The string is made of steel having eight artificial nails attached to right hand and left hand. Music can be performed splendidly via a performer plucks or strums the strings in artificial nails. However, a performer required to understand how to attach each finger to artificial nails and manage the string before the performance. Hence, the cognitive abilities of Zheng players are more crucial than those of keyboard instrument learners.

Previous studies have focused on Zheng instrument practice in learning and performance. For example, most studies investigated the practice problem and effective practice strategies to solve practice problems, and to improve the performance level and achievement on the Zheng (Fu, 2004; Lin, 2008; Mao, 2007; Zhao, 2007; Zhou, 2007). Lin (2008) studied the elements of an effective practice, setting a goal and planning of practice and self-confidence for performing, and monitoring the behavior during practicing processes. In addition, Zhou (2007) found that Zheng instrument has technical problems during performance and practice, including tempo, rhythm, music expression and practice strategies, such as the size of segments played without interruption and the separation of hands, absolute repetitive practice. It also has psychological consequences, such as lack of confidence and intention with a considerable reliance on teachers. The process of Zheng instrument practice is discussed by Zhao (2007) and defined the following phases, preparing phase, planning phase, and performing phase. In addition to Zhao, Fu (2004) emphasized one of the Zheng learning processes which is preparing phase consisting of organizing the structures that are separated, attach the artificial nails, and match the playing posture.

Previous research on Zheng teaching and performance, and practice are among the least researched areas of music education. In light of the above, one way to overcome such issues are to conceptualize the theoretical framework of self-regulation for Zheng musicians. It is necessary to provide guidance on how to approach individual practice to gain the level of success that will able them to reach their potential and to manage and maintain their practice sessions and improve their management ability for effective performance.

\section{Method}

To develop an SRL strategies embedded program of Zheng practice learning programs for novice learners, the following procedures were conducted.

\subsection{Deriving the SRL strategies for the Zheng Practice Program}

We conducted three phases to derive the SRL strategies appropriate for Zheng practice. First, we conducted the instructional analysis to analyze learners, practice problems and SRL strategies during Zheng practice through two types of questionnaire survey with 41 students and 15 Zheng instructors in China, and we also conducted a materials analysis with 4 Zheng books. Second, the "SR Zheng practice viability questionnaire" was constructed based on the instrument analysis and materials analysis, they were also classified with a tool of test self-regulation strategies originally developed by Kim (2009). And finally, the SRL strategies appropriate for Zheng practice were derived by integrating the negative responses (50\% and more) collected from a questionnaire survey with 40 students and an interview with 10 students in China, and some SRL strategies were used most commonly among programs.

\subsection{Making the Zheng Practice Model}

The literature on the literature related to materials of Zhen and music instrument teaching was conducted to find the practice strategies in performance and for education that include the processes of effective practice phases and the features of music instrument practice. We compared different features and the practicing processes between Zheng and other musical instruments, such as piano and organ. Thus, we made the Zhen practice model based on the results of the literature related to materials of Zhen and music instrument teaching. Finally, the expert evaluation of the Zheng practice model was made to identify the content validity of each phase and learning activities of the Zheng practice model which contains two specialists in string music instruments in South Korea. The Zheng practice was made by 
reflecting improvements which were identified by the results of expert evaluation.

\subsection{Developing the Zheng Practice Program Embedded On SRL Strategies}

We developed the Program of Zheng learning based on the Zheng practice model and self-regulated strategies divided previously that consisted of 20 hours for Zheng novices. The self-regulated strategies were applied in each phase. The Zheng Practice Program consisted of 20 periods that include the basing contents of Zheng, self-regulated practice strategies, and Zheng skills. The formative valuation was conducted on three novice learners, and then the Zheng practice program embedded on SRL strategies was developed by expecting the improvements which were identified in the interview phase.

\section{Results and Discussion}

\subsection{Deriving the Components of Self-Regulated Learning}

The SRL strategies appropriate for Zheng practice were derived by integrating the negative responses (50\% and more) collected from a questionnaire survey with 40 students and an interview with 10 students in China, and some SRL strategies are used most commonly among programs. The finding of SRL strategies appropriates for Zheng practice was derived by integrating the cognitive, motivational, and behavior strategies. The cognitive strategies were rehearsal, elaboration, planning and monitoring. The rehearsal factors of Zheng practice include "to repeat and memorize score-aloud", "to repeat and hear the music", "to read aloud repeatedly while drawing by hand the complex part of music", "to perform the whole score once, and then practice repeating the most difficult musical score parts". The elaboration factors of Zheng practice include "easier to say difficult techniques and concepts in the score", "to do score markings, including in the treble, bass, and dynamic range." Planning factors of Zheng practice include "to thinking and determining which part needs to be practiced first or greater repetition", "to plan practice goal, content, order, time before practice implementation", "to divide the whole into relevant parts before implementation". Monitoring factors of Zheng practice include "monitoring the tempo and pace of the musical score", "to monitor whether the planned goal, order, and practice time are effective or ineffective", "to monitor whether my practice is proceeding as planned", "to monitor whether I perform preparation activities properly, or it does or does not include in Zheng constructing, artificial nails attaching, sitting posture, and tuning, To monitor musical score rhythm, tempo, and expression".

The motivational strategies in the Zheng practice program are achieving value, self-efficacy, and mastery goal orientation. The achieving value factors of Zheng practice include "I can express well if repeat reading of the score and understand its atmosphere." "I can express well if we do proper activities well, including in the Zheng constructing, artificial nails attaching, sitting posture, and tuning", "I can practice effectively as planned", "I can improve the performance through frequent evaluation", The self-efficacy factors of Zheng practice include "I can continue the evaluation of my practice during practice and/or after practice", "I can keep practicing well in any music style", "I can control my practice process well during the class or after the class setting", "I can perform effectively in front of audiences", "I can keep recoding my practice every day", "I can appreciate music every day". The mastery goal orientation factors of Zheng practice include "I focus more on understanding the music background than just memorizing the score", "I am satisfied with learning taking place for my practice despite mistakes".

The behavior factors in the ZPP are time management and help-seeking. The time management factors of Zheng practice include "clearing the time, the content, and the goal of practice before starting", "to choose a good and practical practice time", to post the time plan on the practice room", "I make sure to practice as planned, despite experienced negative emotions". The help-seeking factors of Zheng practice includes " to ask friends or a teacher for assistance, advice and find some materials from the internet when encountering score or practice implementation difficulties", "to record my performance and listen to its revision or alteration", "to perform in front of family or acquaintance and get the performance feedback", "to use the metronome in practice when encountering difficulty", "to listen to the music on a CD or a video for determining the atmosphere of the music". 


\subsection{Making The Zheng Practice Model}

The whole illustration of the Zheng practice model is shown as Fig. 1.

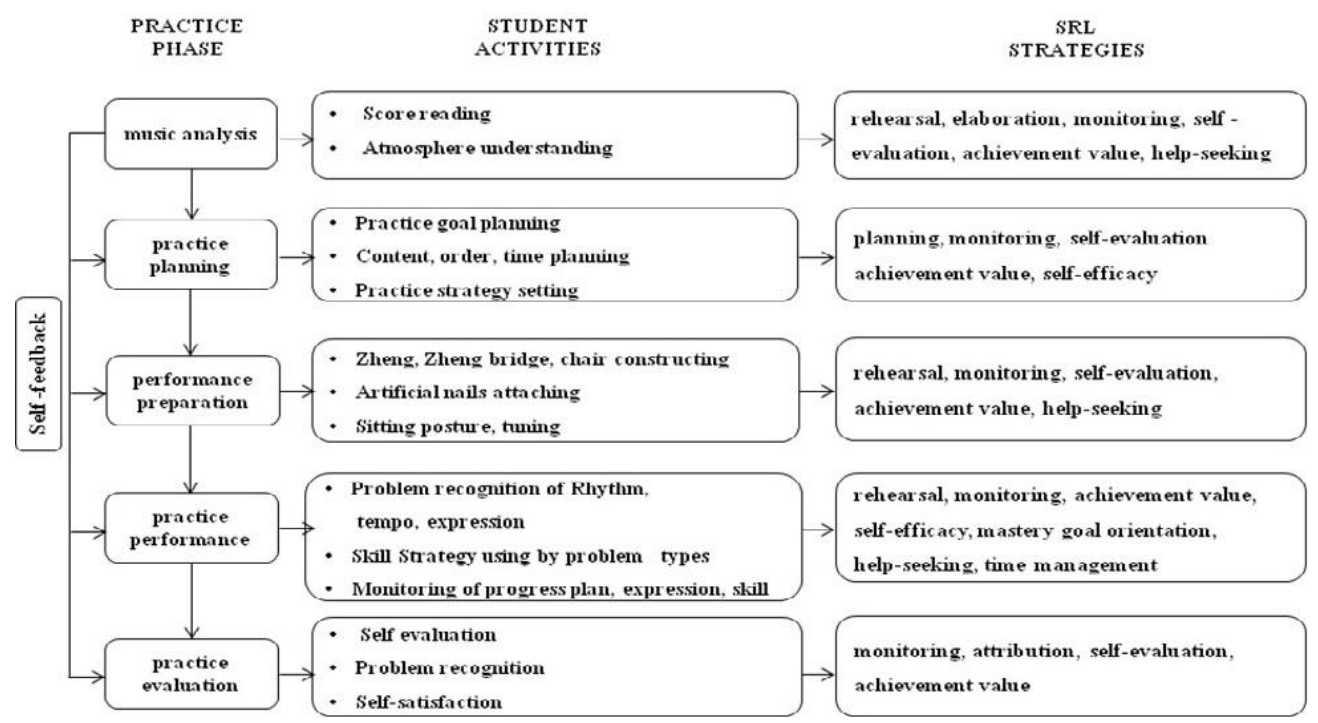

Figure 1. Zheng Practice Model

A Zheng practice model was made from an analysis of literature related to materials of Zheng and music instrument teaching which of music analysis, planning, performance preparation, implementation, and evaluation of practice. After collecting the literature analysis, we found that the analysis of music, planning, preparation, implementation, evaluation of practice was important for effective Zheng practice in which each phase includes different learner activities. The expert evaluation was conducted to identify the content validity for each phase and learning activities of the Zheng practice model. Content validity of the Zheng practice model was constructed as 4.5 by expert evaluation. The result of the usability test showed that the mean score of the survey was 4.5. Finally, the Zheng practice model was constructed by reflecting improvements which were identified by questionnaire.

\subsection{Developing the Zheng Practice Program Embedded On SRL Strategies}

The Program of Zheng learning was developed that consisted of 20 periods for Zheng novices, including Zheng contents (1st-5th period), SRL strategies appropriate for Zheng practice (6th period), and Zheng skills (7th-20th period). The worksheet was used in each period that includes a score analysis table, a planning table of practice, a performance checklist, a self- evaluation table for daily practice, and practice reflection table for one week used in each practice phase. Formative valuation was conducted on 3 novice learners for the developed ZPP based on SRL strategies for 5 weeks. Finally, the developed ZPP reflected improvements which were identified via an interview. It can enable novice music learners to engage their practice processes and improve their management ability for effective music practice.

The components of SRL for the ZPP that employed in the Zheng practice program are derived in three dimensions of cognitive regulation, motivation regulation and behavioral regulation. These were primary factors in the practical strategies of self-regulation. The present study involved analysis and identification of effective components of music instrument practice; investigated students' use of SRL strategies in practice and examined their relationship with respect to SRL and music instrument practice processes (Chung, 2006; 2007; Hallam, 2001; McPherson \& Renwick, 2001; Nilesen, 1999; 2001). In particular, this study reviewed meta-cognition factors during instrument practice. A relathioship between music instrument practice and SRL was revealed. However, it is rarely used appropriate SRL strategies applied to music instrument practice. Thus, this study is meaningful for effective practice in the musical field.

A Zheng practice model was constructed in the analysis of music, planning, preparation, implementation, evaluation of practice which is critical for the effective Zheng practice. The analysis of the score and performance preparation phase are different from other studies (Hallam, 1997; Nielson, 2001). And the music instrument practice model is rarely developed and used in the previous research. In this study, the music analyzing phase is used to score reading an understanding of its atmosphere. The performance preparation involves Zheng's instruction, artificial nails attachment, sitting posture, and tuning. Hence, a music instrument practice model needs to consider the nature of the musical instrument, and the effective practice phase and learning activities pertinent to different kinds of musical instruments such as keyboard and string music instruments. The Zheng practice model provides a practical guideline for the Zheng 
novice learner.

The Zheng practice program was developed by incorporating different learning activities in regard to each practice phase based on SRL strategies that are appropriate. It is meaningful for the provision of a systematic practice phase within the Zheng teaching situation which doesn't consider practice important. Furthermore, the Zheng program is improved through a formative valuation of 3 novice learners, but the validity evaluation couldn't be conducted. This content validity of the Zheng practice program is necessary for the future direction conduct of research.

\section{Conclusions}

An effective music practice of a music instrument can be achieved through the use of self-regulated learning strategies. In this study, we developed a program of Zheng learning for novice learners to Zheng practice by using SRL strategies. Individual music practices are crucial for student musicians in order to improve their performance skills and optimistic to become professional musicians. It can allow more learner control and require learners to take responsibility of their own practice.

SRL strategies appropriate for Zheng practice which have the potential to promote the learner's individual practice were derived by integrating the negative responses collected from a questionnaire survey with 40 students and an interview with 10 students in China, and some SRL strategies are used most commonly among program. The finding of SRL strategies appropriates for Zheng practice was derived by integrating the cognitive, motivational, and behavior strategies. The cognitive strategies appropriate for Zheng practice were rehearsing, elaboration, planning, and monitoring. The motivational strategies appropriate for Zheng practice were achievement value, self-efficacy, and mastery goal orientation. The behavior strategies appropriate for Zheng practice were time management and help-seeking.

A Zheng practice model was made from an analysis of literature related to materials of Zheng and music instrument teaching which are music analysis, planning, performance preparation, implementation, and evaluation of practice. On the basis of the Zheng practice model, program of Zheng learning was developed that consisted of 20 periods for Zheng novices, including Zheng contents (1st-5th period), SRL strategies appropriate for Zheng practice (6th period), and Zheng skills (7th-20th period). The worksheet was used in each period that includes an analysis table of score, planning table of practice, a checklist for performing, self-evaluating table for practice, practice daily, and practice reflection table for one week used in each phase of practice.

\section{References}

Chaffin, R., \& Imreh, G. (2001). A comparison of practice and self-report as sources of information about the goals of expect performance. Psychology of Music, 29, 39-69.

Chung, J. W. (2006). Self-regulated learning in piano practice of middle school piano majors in Korea. Doctoral dissertation. Columbia University.

Ericsson, K. A., Krampe, R. T., \& Tesch-Romer, C. (1993). The Role of deliberate practice in the acquisition of expert performance. Psychological Review, 100(3), 363-406.

Fu, M. J. (2004). Zheng Learning, Practicing, Performing. Journal of Qin Zheng.

Hallam, S. (2001). The development of expertise in young musicians: Strategy use, knowledge acquisition, and individual diversity. Music Education Research, 3(1), 7-23.

Kim, L. Y. (2009). A study on the self-regulation of learners in practicing the piano. Doctoral dissertation. Korea National University of Education.

Leon-Guerrero, A. J. (2008). Self-regulation strategies used by student musicians during music practice. Music Education Research, 10(1), 91-106

Lin, L. (2008). Basic Zheng textbook. People's Music Publishing House.

McPherson, G. E., \& Renwick, J. M. (2001). A longitudinal study of self-regulation in children's musical practice. Music Education Research, 3, 169-186.

Miksza, P. (2007). Effective practice: An investigation of observed practice behaviors, self-reported practice habits, and the performance achievement of high school wind players. Journal of Research in Music Education, 55,359-375. http://dx.doi.org/10.1177/0022429408317513.

McPherson, G. E., \& Renwick, J. M. (2006). Self-efficacy and music performance. Psychology of Music, 34, $322-336$. Mao, J. (2007). How to improve the effect on Zheng's performing. Journal of Teachers College Nan Yang.

McPherson, G. E., \& Zimmerman, B. J. (2002). Self-regulation of musical learning. R. Colwell \& C. Richardson(Eds.), 
The New Handbook of Research on Music Teaching and Learning. New York: Oxford University Press., 327-347.

Nielsen, S. G. (1999). Regulation of learning strategies during practice: A case study of a single church organ student preparing a particular work for a concert performance. Psychology of Music and Music Education, 27, $218-229$.

Nielsen, S. G. (2001). Self-regulating learning strategies in instrumental music practice. Music Education Research, $3(2), 155-167$.

Nielsen, S. G. (2004). Strategies and self-efficacy beliefs in instrumental and vocal individual practice: a study of students in higher music education. Psychology of Music. 32(4), 418-431

Pitts, D. A., \& McPherson, G. (2000). Developing effective practice strategies: Case studies of three young players in the first 20 months of learning. Music Education, 146, 51-69.

Miao, S., \& Samuel, W. (2005), Qi: An Instrumental Guide to the Chinese Orchestra. Singapore: Teng.

Smith, B. P. (2002). The role of selected motivational beliefs in the process of collegiate instrumental music practice. Doctoral dissertation. University of Michigan.

Susan, J. K. (2008). A collective case study of self-regulated learning in instrumental practice of college music majors. Doctoral dissertation. Columbia University.

Williamon, A., \& Valentine, E. (2000). Quantity and quality of music practice as predictors of performance quality. British Journal of Psychology, 91(3), 353-376.

Zhao, Y. (2007). The Changes in Zheng skill and the problems in Zheng teaching. Journal of Wuhan, China Music, 2 , J632.32.

Zimmerman, B. J., \& Martinez-Pons, M. (1986). Development of a structure interview for assessing student of self-regulated learning strategies. American Educational Research Journal, 23(4), 614-628.

Zimmerman, B. J. (2000). Attaining self-regulation: A social cognitive perspective. In M. Boekaerts, P.R. Pintrich, \& M. Zeidner (Eds.), The handbook of self-regulation. San Diego, CA: Academic Press, 13-39.

Zimmerman, B. J. (1986), Becoming a self-regulated learner: Which are the key subprocesses? Contemporary Educational Psychology, 11, 307-313.

Zhou, W. (2007). Zheng learning. Classic Pieces of Zheng. Beijing TT-ART Publishing House, China.

\section{(cc) $\mathrm{Br}$}

This work is licensed under a Creative Commons Attribution 3.0 License. 